Article

\title{
Fructose-Induced Intestinal Microbiota Shift Following Two Types of Short-Term High-Fructose Dietary Phases
}

\author{
Julia Beisner ${ }^{\dagger}$, Anita Gonzalez-Granda ${ }^{\dagger}$, Maryam Basrai, Antje Damms-Machado \\ and Stephan C. Bischoff * \\ Institute of Nutritional Medicine, University of Hohenheim, Fruwirthstr. 12, 70599 Stuttgart, Germany; \\ julia.beisner@uni-hohenheim.de (J.B.); anita.gonzalez@hospitalgonzalez.ec (A.G.-G.); \\ m.basrai@uni-hohenheim.de (M.B.); antje.damms@googlemail.com (A.D.-M.) \\ * Correspondence: bischoff.stephan@uni-hohenheim.de \\ + These authors contributed equally to this work.
}

Received: 1 October 2020; Accepted: 5 November 2020; Published: 10 November 2020

\begin{abstract}
High consumption of fructose and high-fructose corn syrup is related to the development of obesity-associated metabolic diseases, which have become the most relevant diet-induced diseases. However, the influences of a high-fructose diet on gut microbiota are still largely unknown. We therefore examined the effect of short-term high-fructose consumption on the human intestinal microbiota. Twelve healthy adult women were enrolled in a pilot intervention study. All study participants consecutively followed four different diets, first a low fructose diet $(<10 \mathrm{~g} /$ day fructose), then a fruit-rich diet (100 g/day fructose) followed by a low fructose diet (10 g/day fructose) and at last a high-fructose syrup (HFS) supplemented diet (100 g/day fructose). Fecal microbiota was analyzed by $16 \mathrm{~S}$ rRNA sequencing. A high-fructose fruit diet significantly shifted the human gut microbiota by increasing the abundance of the phylum Firmicutes, in which beneficial butyrate producing bacteria such as Faecalibacterium, Anareostipes and Erysipelatoclostridium were elevated, and decreasing the abundance of the phylum Bacteroidetes including the genus Parabacteroides. An HFS diet induced substantial differences in microbiota composition compared to the fruit-rich diet leading to a lower Firmicutes and a higher Bacteroidetes abundance as well as reduced abundance of the genus Ruminococcus. Compared to a low-fructose diet we observed a decrease of Faecalibacterium and Erysipelatoclostridium after the HFS diet. Abundance of Bacteroidetes positively correlated with plasma cholesterol and LDL level, whereas abundance of Firmicutes was negatively correlated. Different formulations of high-fructose diets induce distinct alterations in gut microbiota composition. High-fructose intake by HFS causes a reduction of beneficial butyrate producing bacteria and a gut microbiota profile that may affect unfavorably host lipid metabolism whereas high consumption of fructose from fruit seems to modulate the composition of the gut microbiota in a beneficial way supporting digestive health and counteracting harmful effects of excessive fructose.
\end{abstract}

Keywords: fructose; microbiota; host-microbe interactions; obesity; metabolic syndrome; NAFLD

\section{Introduction}

The prevalence of overweight and obesity is increasing worldwide. The World Health Organization (WHO) reports that in 2016 39\% of adults (> 18 years) were overweight and 13\% were obese. The prevalence of overweight children and adolescents has increased dramatically from $4 \%$ in 1975 to $18.5 \%$ in 2016 . Changes in dietary and eating behavior such as consumption of sugar-sweetened beverages and sugar-rich processed food high in fat and refined carbohydrates, the so-called western 
style diet (WSD) in conjunction with lower levels of physical activity are associated with a rise in obesity [1]. Among the dietetic influences, particularly sucrose- and fructose-rich soft drinks are the most critical factors causing the development of obesity and fatty liver disease.

Fructose naturally appears in fruits and vegetables, but it is also often used as a cheap, refined carbohydrate sweetener in the form of high-fructose-glucose syrup for soft drinks, sweets and highly processed foods. Although the clinical impact of long term high-fructose consumption is still controversial [2], many studies have shown the harmful effects of a high-fructose intake in animal models [3-6] and humans [7-11]. High-fructose consumption may be associated with obesity [12], metabolic syndrome [13] and non-alcoholic fatty liver disease (NAFLD) [14-17]. Although the exact mechanism of fructose induced development of NAFLD is still not fully understood [14], it is known that a high-fructose consumption causes epithelial barrier dysfunction by increasing intestinal permeability [18-20]. Consequently, endotoxins like lipopolysaccharides (LPS) can translocate through the mucosa into the blood stream leading to metabolic endotoxemia $[16,21,22]$.

Diet is one of the various factors that influences the gut microbiota composition [14,23]. Dietary fructose and glucose, which are prevalent in the Western diet, affect host-gastrointestinal microbe interactions contributing to differences observed in obese and normal-weight intestinal microbiota. However, only a few studies investigated the impact of fructose-rich diets on gut microbiota and the subsequent effects of high-fructose diet-induced effects on metabolic diseases. A high-fructose or a high-sugar consumption has been shown to influence the microbiota composition $[24,25]$ and alters the abundance of Bacteroidetes and Firmicutes [6,26,27]. In rats fed a fructose-rich diet, the development of metabolic syndrome was correlated with variations of the gut content of specific bacterial genera [28]. Crescenzo et al. [29] reported that a fructose-rich diet promoted alterations in the gut microbiota profile in rats and, moreover, that these alterations were associated with inflammation and metabolic dysregulation in the gut and liver. The substantial link between microbiota dysbiosis and NAFLD has been supported by results from animal studies showing that obesity-related features in fructose-fed rats were reversed by changes in the gut microbiota profile and development of NAFLD was markedly reduced by treatment with antibiotic therapy, prebiotics and selected probiotics [28-30]. The fructose-induced microbiota alteration is therefore considered a critical factor contributing to NAFLD progression in animal models and humans and has been associated with the metabolic syndrome [31].

The aim of the present study was to investigate the influence of different types of short-term fructose-rich diets on the human gut microbial signature. Therefore, we analyzed the human microbiota composition of healthy females following consecutively different high-fructose diets, first a fruit diet (100 g/day fructose from fruit and vegetables) and then, after a low-fructose phase, a HFS diet (100 g/day fructose from syrup) and characterized the responses of bacterial communities in the study subjects that underwent the dietary intervention. In a previous study, we found significant changes especially in certain acylcarnitine and lysophosphatidylcholine levels after high-fructose consumption in the same study cohort [32] and therefore also correlated the microbiota abundances with plasma metabolites. Here we report a shift in the bacterial abundances after high-fructose diets.

\section{Materials and Methods}

\subsection{Study Design}

This pilot study is an open-label, single-arm intervention study and included six lean and six obese healthy volunteers, who underwent four different diets. During the first week, study subjects received a low-fructose diet (low f1), which served as control diet. The second week consisted of a high-fructose diet rich in fruits and vegetables (fruit). During the third week subjects again followed a low fructose diet (low f2), which was identical to low f1 and served as wash-out phase. During the fourth week, study subjects received a high-fructose diet supplemented with high-fructose syrup (HFS). The study design is graphically shown in Figure 1. 


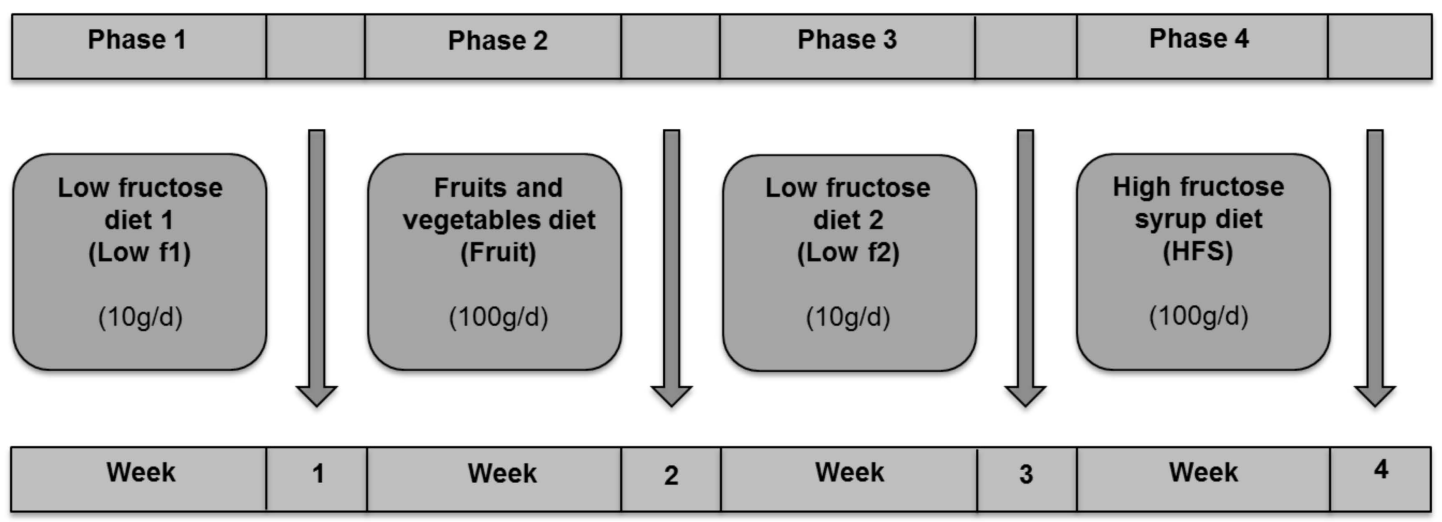

Figure 1. Study design. Study subjects received a low-fructose diet 1 (low f1), a fruits and vegetables diet (fruit), a low fructose diet 2 (low f2) and a high-fructose syrup diet (HFS).

\subsection{Selection of Subjects}

Twelve healthy female volunteers, aged between 20 and 40 years, were recruited through email distribution from the University of Hohenheim, Germany, over a time period of six weeks. After written informed consent, a medical history, physical examination, blood sample collection and a hydrogen breath test were performed to exclude fructose intolerance before enrollment to ensure eligibility. The subjects had to be non-smokers, and non-pregnant/non-breastfeeding. Women with chronic gastrointestinal diseases who have undergone gastrointestinal surgery (other than appendectomy), women with chronic anemia, chronic hepatic or renal disease, diabetes mellitus or other relevant chronic health disorders or regular medication were not included in the study. Subjects who passed the eligibility criteria were allocated according to their body mass index (BMI) either to the lean $(20<$ BMI $\left.<25 \mathrm{~kg} / \mathrm{m}^{2}, n=6\right)$ or to the obese $\left(35<\mathrm{BMI}<50 \mathrm{~kg} / \mathrm{m}^{2}, n=6\right)$ group. Table 1 shows the baseline characteristics of the study population. The study was approved by the responsible ethics committee (Landesärztekammer Baden-Württemberg, Stuttgart, Germany; no. 2009-079-f) in 2009, registered at ClinicalTrials.gov (ID: NCT03444233) and carried out in accordance with the Helsinki Declaration (revised version, 1989).

Table 1. Baseline characteristics of the study population.

\begin{tabular}{cccc}
\hline Characteristics & $\begin{array}{c}\text { Lean } \\
(\boldsymbol{n}=\mathbf{6})\end{array}$ & $\begin{array}{c}\text { Obese } \\
(\boldsymbol{n}=\mathbf{6})\end{array}$ & $\boldsymbol{p}$-Value \\
\hline Age (years) & $26 \pm 2$ & $30 \pm 3$ & 0.013 \\
BMI (kg/m $\left.{ }^{2}\right)$ & $22.5 \pm 1.5$ & $41.5 \pm 4.0$ & 0.004 \\
WC (cm) & $72.4 \pm 2.5$ & $118.3 \pm 9.4$ & 0.004 \\
BP sys (mmHg) & $104.2 \pm 7.4$ & $122.5 \pm 9.9$ & 0.004 \\
BP dias (mmHg) & $69.2 \pm 6.7$ & $83.3 \pm 6.8$ & 0.005 \\
FBG (mg/dL) & $87.7 \pm 4.6$ & $93.3 \pm 8.4$ & $\mathrm{~ns}$ \\
HDL (mg/dL) & $62.0 \pm 11.4$ & $53.8 \pm 14.2$ & $\mathrm{~ns}$ \\
LDL (mg/dL) & $100.0 \pm 22.1$ & $137.2 \pm 35.4$ & 0.025 \\
TG (mg/dL) & $82.0 \pm 20.5$ & $108.7 \pm 63.7$ & $\mathrm{~ns}$ \\
FLI & $7.0 \pm 2.5$ & $89.8 \pm 12.5$ & 0.001 \\
HRI & $0.9 \pm 0.2$ & $1.0 \pm 0.1$ & $\mathrm{~ns}$ \\
GGT & $15.5 \pm 2.2$ & $25.5 \pm 17.7$ & $\mathrm{~ns}$ \\
ALT & $16.2 \pm 3.1$ & $23.7 \pm 6.2$ & 0.024 \\
\hline
\end{tabular}

Data are expressed as mean ( \pm SD). Abbreviations: ALT, alanine aminotransferase; BMI, body mass index; BP sys, systolic blood pressure; BP dias, diastolic blood pressure; FBG, fasting blood glucose; FLI, fatty liver index; GGT, gamma-glutamyl-transpeptidase; HDL, high-density lipoproteins; HRI, hepatorenal index; LDL, low-density lipoprotein; NW, normal weight; $\mathrm{OB}$, obese; TG, triglycerides; WC, waist circumference. 


\subsection{Dietetic Intervention}

Before study intervention, all subjects participated in a nutritional training, in which they were informed about the sugar content in foods. Additionally, each subject received an individual nutritional counselling based on an individualized nutritional plan.

The previewed diets were largely isocaloric and isonitrogenous with $30 \%$ of total energy intake derived from fat, $15 \%$ from protein and 55\% from carbohydrates. The fructose content during the low-fructose diet phases was less than $2 \%$ of the caloric intake. During the high-fructose diet phases, the fructose content was set to around $20 \%$ of total energy intake. The energy need was individually calculated using the Harris-Benedict-equation $655+(9.56 \times$ weight $(\mathrm{kg})+(1.85 \times$ height $(\mathrm{m})-4.68$ $\times$ age $($ years $)) \times 1.5$. A physical activity level of 1.5 was subjected to all patients since none of them reported extreme physical activity or decreased activity below normal. Body weight was determined weekly during the intervention and was also used to check energy balance. The diets and the nutrient intake of the study population have previously been described in more detail [32]. After study inclusion, the subjects underwent the four diet phases (Figure 1).

During the first week, subjects underwent the first low fructose diet phase (lowf1). In this diet phase, subjects had to avoid sweets, highly processed foods, soft drinks and fruits and vegetables containing more than $1 \mathrm{~g}$ fructose/100 g. A fructose uptake of up to $10 \mathrm{~g} /$ day was tolerated. During the second week, subjects followed a high-fructose diet rich in fruits and vegetables (fruit) corresponding to a fructose intake of about $100 \mathrm{~g} /$ day. Highly processed foods, sweets and sweetened soft drinks had to be avoided to ensure a low level of free fructose uptake dissolved in beverages or derived from sugar syrups. The third diet week was again a low-fructose diet phase (lowf2) equal to lowf1. The fourth diet phase was a high-fructose diet phase achieved by supplementation with high-fructose syrup (HFS). In this phase, the subjects were asked to sweeten their meals with $100 \mathrm{~g} /$ day fructose-glucose-syrup from corn containing 40-44\% fructose (C-TruSweet 01750, Cargill Deutschland GmbH, Krefeld). Fructose-rich foods like fruits and juices had to be avoided.

A daily dietary record was conducted by the study subjects to assess the actual nutritional intake and was analyzed using the EBISPro software, version 8.0 (Dr. Erhardt, University of Hohenheim, Stuttgart, Germany). According to these records, study participants varied their energy intake significantly within the different diet phases. During the fruit-rich diet phase, energy intake increased by about $25 \%$ and during the HFS diet phase by about $50 \%$. Table 2 shows the energy and nutrient intake of the different diets. The mean nutritional intake of study subjects was described in more detail elsewhere [32]. In brief, during the low-fructose diet phases, subjects consumed higher amounts of fat compared to the defined target. The fruit-rich diet was characterized by a significantly higher intake of fiber compared to the lowf1, lowf2 and HFS diets. To reach a fructose uptake of $100 \mathrm{~g} / \mathrm{day}$, high amounts of fruits and vegetables had to be consumed. In fact, sweet fruits like grapes were the preferred choice to reach the defined fructose target of $100 \mathrm{~g} /$ day rather than fiber-rich vegetables. During the HFS phase, the energy intake was higher compared to the defined target. This can be explained by the consumption of the fructose-glucose syrup of $100 \mathrm{~g} /$ day additionally to the normal diet. 
Table 2. Composition of study diets.

\begin{tabular}{|c|c|c|c|c|c|c|}
\hline \multirow[t]{2}{*}{ Nutrients } & \multicolumn{2}{|c|}{ Low Fructose Diet } & \multicolumn{2}{|c|}{ Fruits } & \multicolumn{2}{|c|}{ HFS } \\
\hline & Goal & Actual Intake & Goal & Actual Intake & Goal & Actual Intake \\
\hline \multicolumn{7}{|c|}{ Energy (kcal/day) } \\
\hline NW & $2002 \pm 56$ & $1828 \pm 260$ & $2005 \pm 54$ & $2188 \pm 357$ & $2006 \pm 59$ & $2404 \pm 404 *$ \\
\hline OB & $2207 \pm 51$ & $1949 \pm 442$ & $2209 \pm 51$ & $2404 \pm 410$ & $2210 \pm 51$ & $2961 \pm 273 *$ \\
\hline \multicolumn{7}{|c|}{ Protein (g/day) } \\
\hline NW & $73 \pm 2$ & $83 \pm 10$ & $73 \pm 2$ & $69 \pm 15$ & $73 \pm 2$ & $65 \pm 10$ \\
\hline OB & $81 \pm 2$ & $102 \pm 41 *$ & $81 \pm 2$ & $91 \pm 29$ & $81 \pm 2$ & $92 \pm 28$ \\
\hline \multicolumn{7}{|c|}{ Fat (g/day) } \\
\hline NW & $65 \pm 2$ & $80 \pm 10 *$ & $65 \pm 2$ & $67 \pm 20$ & $65 \pm 2$ & $70 \pm 20$ \\
\hline $\mathrm{OB}$ & $71 \pm 2$ & $89 \pm 25 *$ & $71 \pm 2$ & $79 \pm 24$ & $71 \pm 2$ & $93 \pm 18$ \\
\hline \multicolumn{7}{|c|}{$\mathrm{CHO}(\mathrm{g} /$ day $)$} \\
\hline NW & $268 \pm 8$ & $176 \pm 52 *$ & $271 \pm 5$ & $308 \pm 45$ & $271 \pm 5$ & $357 \pm 58$ \\
\hline OB & $296 \pm 7$ & $174 \pm 24 *$ & $288 \pm 4$ & $314 \pm 24$ & $288 \pm 4$ & $416 \pm 47$ \\
\hline \multicolumn{7}{|c|}{ Fiber (g/day) } \\
\hline NW & medium & $17 \pm 6$ & Medium & $36 \pm 8$ & low & $12 \pm 5$ \\
\hline $\mathrm{OB}$ & medium & $19 \pm 8$ & Medium & $39 \pm 7$ & low & $19 \pm 7$ \\
\hline \multicolumn{7}{|c|}{ Complex CHO (g/day) } \\
\hline NW & $258 \pm 8$ & $160 \pm 48 *$ & $171 \pm 5$ & $128 \pm 5^{*}$ & $171 \pm 5$ & $121 \pm 30$ * \\
\hline OB & $286 \pm 7$ & $166 \pm 19 *$ & $188 \pm 4$ & $131 \pm 3^{*}$ & $188 \pm 4$ & $174 \pm 32$ \\
\hline \multicolumn{7}{|c|}{ Fru (g/day) } \\
\hline NW & 10 & $7 \pm 2$ & 100 & $100 \pm 12$ & 100 & $106 \pm 11$ \\
\hline OB & 10 & $4 \pm 3$ & 100 & $102 \pm 4$ & 100 & $108 \pm 6$ \\
\hline \multicolumn{7}{|c|}{ Glu (g/day) } \\
\hline NW & low & $7 \pm 2$ & Medium & $80 \pm 8$ & High & $130 \pm 12$ \\
\hline $\mathrm{OB}$ & low & $5 \pm 3$ & Medium & $80 \pm 9$ & High & $134 \pm 6$ \\
\hline
\end{tabular}

Data are expressed as mean $( \pm \mathrm{SD}) .{ }^{*}$ More than $20 \%$ difference between the proposed amount and the actual intake. Abbreviations: $\mathrm{CHO}$, carbohydrates; Fru, fructose; Glu, glucose; HFS, high-fructose syrup; NW, normal weight; $\mathrm{OB}$, obese; actual intakes of fructose and glucose calculated include the amounts of fructose and glucose bound in sucrose.

\subsection{Clinical Study Parameters}

Clinical laboratory parameters fasting glucose, alanine aminotransferase (ALT), gamma-glutamyl-transpeptidase (GGT), alkaline phosphatase (AP), high-density lipoprotein (HDL), low-density lipoprotein (LDL), triglycerides (TG), blood sedimentation rate (BSR), creatinine, urea, and uric acid were assessed. Anthropometric and clinical data (body mass index (BMI), waist circumference (WC) and blood pressure) were determined. Liver ultrasound was performed by a trained physician using the LOGIQ P6 device (GE Healthcare, Solingen, Germany) as described [33]. Hepatorenal index (HRI) was determined according to Webb et al. [34], and fatty liver index (FLI) was assessed according to Bedogni et al. [35]. Plasma metabolites were analyzed by targeted metabolomics [32].

\subsection{Microbiota Analysis by $16 S$ rRNA Amplicon Sequencing}

Stool samples were collected weekly after each diet period in a stool sample vessel with DNA stabilizer. The microbial DNA was isolated using the Invitek PSP-Spin Stool DNA Plus Kit with lysis enhancer according to the manufacturer's instructions (Stratec Molecular, Berlin, Germany). $16 \mathrm{~S}$ amplicon sequencing was performed by CeMet $\mathrm{GmbH}$, Center for Metagenomics, Tübingen. Twenty-five nanogram of genomic DNA was used to prepare amplicon libraries using Nextera XT Index Kit (Illumina, San Diego, CA, USA), according to manufacturer's instruction. Primers targeted the V3-V4 region of the 16S rRNA gene [36]. Paired-end sequencing was performed on an Illumina MiSeq platform (IIIumina, San Diego, CA, USA) using v2 reagents. Sequence reads were processed by a bioinformatic pipeline. PRINSEQ-lite was used to trim sequences by base quality [37]. Subsequently quality control of trimmed sequences was performed by FastQC [38]. Fastq-Join was used for merging trimmed sequences [39]. Merged reads with a length shorter $100 \mathrm{bp}$ were filtered and FastQC was applied again. Taxonomic classification of the sequence data was performed against the National Center 
for Biotechnology Information (NCBI) bacterial 16S rRNA database using MALT [40]. For further analysis, sequence data were analyzed using MEGAN6 software [41] MEGAN6 was used to calculate the bacterial abundance and the Shannon's diversity index of the volunteers. Normalized read counts were used for further statistical analysis. The functional annotation of the reads was done based on the KEGG library (Kyoto Encyclopedia for Genes and Genomes, http://www.genome.jp/kegg/).

\subsection{Statistical Analysis}

Statistical analysis of 16S rRNA gene amplicon sequencing was performed using R software 3.5.1 (R Core Team, Vienna, Austria) and its packages Hotelling, reshape 2, bindr, ggplot2 and heatmap3 as well as RStudio version 1.1.456 (RStudio, Inc., Boston, MA, USA). GraphPad Prism version 7.0 software (GraphPad Software, Inc., La Jolla, USA) was used for graphical presentation of bacterial abundances as well as for statistical analyses. Laboratory, clinical and nutritional parameters were tested for normal distribution using Kolomogov-Smirnov test. In case of normally distributed data unpaired Student's $t$-test was used to test for significant differences between group means. Mann-Whitney U test was used to test for differences between group means in case of non-normally distributed data. Mann-Whitney $U$ test was performed for analyzing differences in microbiota abundance between lean and obese subjects. Differences within diet phases were analyzed using paired Student's $t$-test with Bonferroni correction for multiple testing. Significant changes between phases in microbiota abundance were analyzed using Wilcoxon signed-rank test with Benjamini-Hochberg (false discovery rate (FDR)) correction for multiple comparisons. Pearson's correlation analysis was performed for analyzing correlations between clinical and anthropometric parameters and microbiome data. $p$-values of $<0.05$ were considered as statistically significant.

\section{Results}

\subsection{Gut Microbiota Profile in Study Subjects}

The most abundant phyla were Firmicutes accounting for $52.6 \%$ of abundance at phylum level, Bacteroidetes for $36.0 \%$, Proteobacteria for $5.7 \%$, Actinobacteria for $3.2 \%$ and Verrucomicrobia for $2.3 \%$ (Figure 2B). The remaining phyla accounted for $0.2 \%$. At the genus level Bacteroides clearly dominated (28.4\%), followed by Faecalibacterium (6.4\%), Ruminococcus (3.6\%), Alistipes (3.3\%) and Bifidobacterium (2.9\%). During all phases the Firmicutes abundance clearly dominated (lowf1, 52.6\%; fruit, 61.6\%; lowf2, 51.6\%; HFS, 48.8\%), followed by Bacteroidetes (lowf1, 36.0\%; fruit, 32.1\%; lowf2, 39.9\%; HFS, 43.1\%), Proteobacteria (lowf1, 5.7\%; fruit, 3.5\%; lowf2, 4.6\%; HFS, 3.6\%) and Actinobacteria (lowf1, 3.2\%; fruit, 2.1\%.; lowf2, 2.8\%; HFS, 3.6\%). Among the different diet phases lowf1, fruit, lowf2 or HFS changes in the abundances of bacterial taxa were observed (Figure 2A). The bacterial diversity did not change significantly within the phases, although a minor pattern was marginally visible namely higher indices after fructose-poor diets (lowf1, lowf2) and lower ones after fructose-rich diets (fruits, HFS; data not shown). 
A

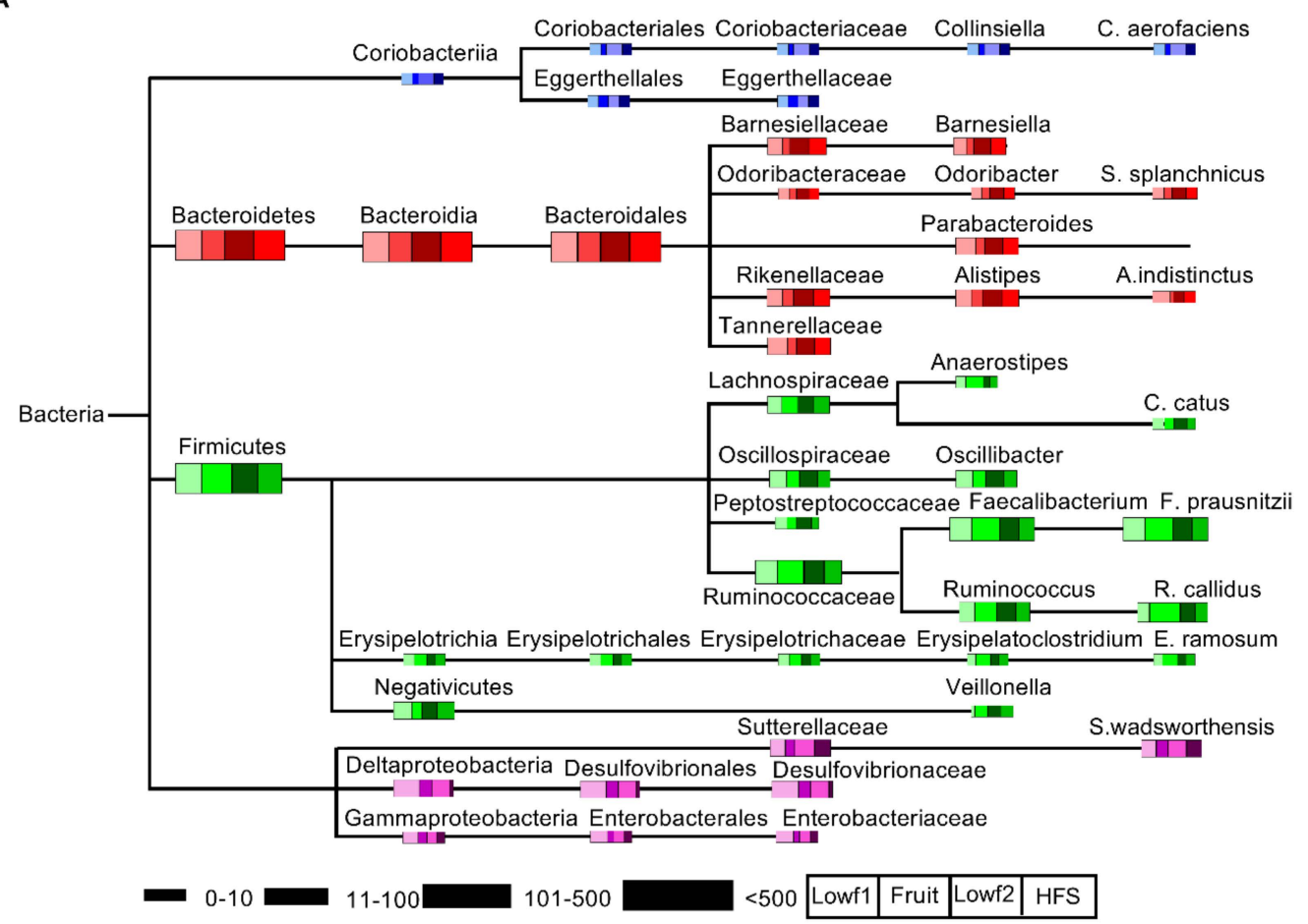

B

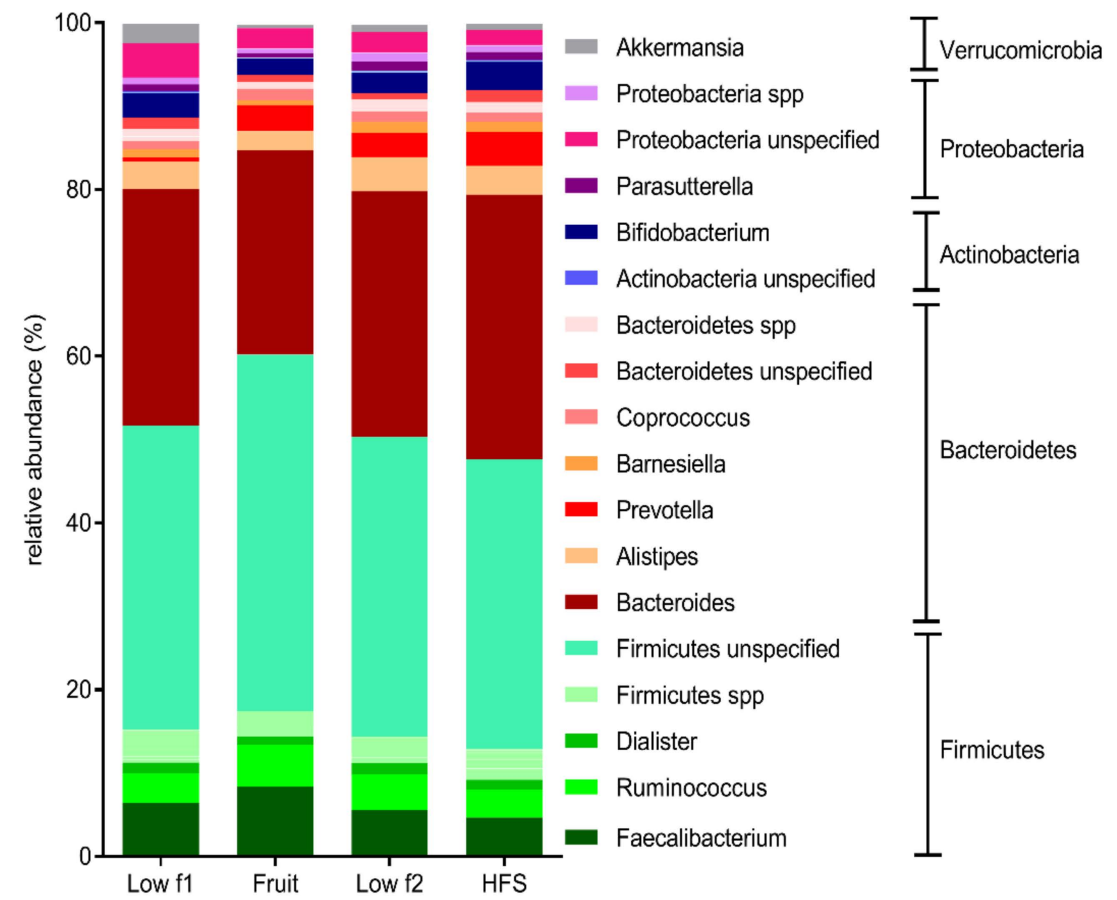

Figure 2. Effects of different fructose diets on the composition of the gut microbiome. (A) Composition of bacterial communities that changed among the different fructose diets. The schematic dendogram was constructed from $16 \mathrm{~S}$ rRNA sequencing analysis results. Size of the bars represents the abundances of the bacterial taxa. Different phyla were shaded by different colors: blue, Actinobacteria; red, Bacteroidetes; green, Firmicutes; purple, Proteobacteria. (B) Distribution of the relative abundances at phylum and genus level between the different fructose diets. 


\subsection{Fructose Dependent Changes of Relative Abundance on Phylum Level}

At the phylum level, high-fructose diets induced differences in microbiota composition, especially changes in Firmicutes and Bacteroidetes abundance. Relative abundance of Firmicutes was increased after the fruit diet and decreased when study participants switched from the fruit to the lowf 2 and HFS diet, whereas the relative abundance of Bacteroidetes showed opposing patterns suggesting a fructose-dependent modulation of the gut microbiome (Figure 3). The Firmicutes abundance changed significantly from the fruit to lowf2 diet phase (FDR-adjusted $p=0.021$ ), whereas changes in Bacteroidetes abundance were not significant after FDR correction $(p=0.075)$. Comparing the fruit and HFS diet phases, significant differences were observed in Firmicutes and in Bacteroidetes abundance (Figure 3A). Analyzing the subgroup of obese subjects separately, a similar pattern in microbiota changes was observed (Figure 3B), although the changes in Firmicutes and in Bacteroidetes abundance were only significant without correction for multiple testing. The Firmicutes to Bacteroidetes ratio is regarded to be of significant relevance in human gut microbiota composition. In the Firmicutes/Bacteroidetes ratio, we observed significant differences between the fructose-rich diets. The ratio of Firmicutes to Bacteroidetes (F/B ratio) was significantly decreased after the HFS diet compared to the fruit diet (Figure 3C).
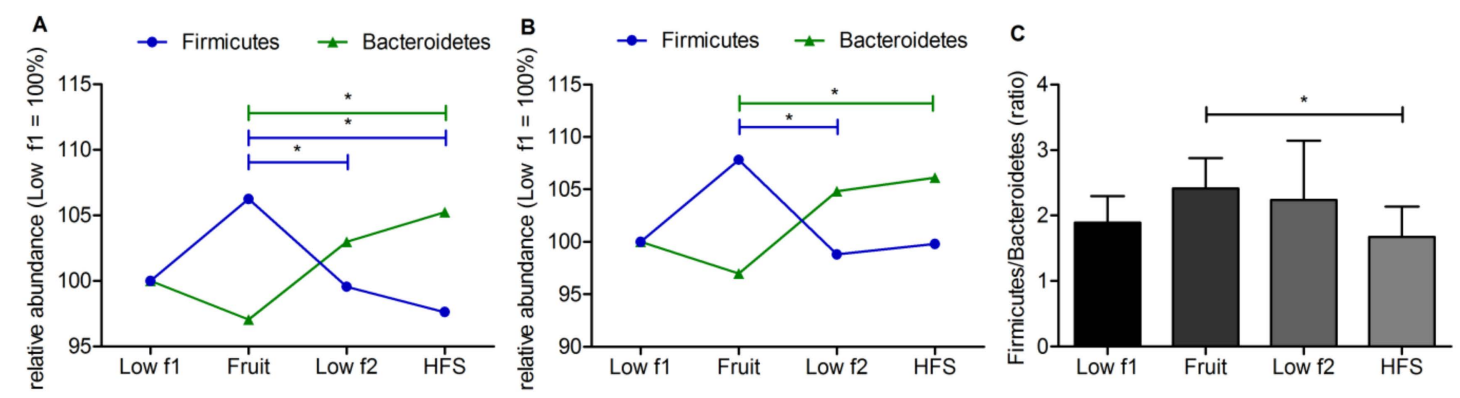

Figure 3. Significant changes between fructose diet phases on phylum level. Relative abundance of Bacteroidetes and Firmicutes in all $(n=12)(\mathbf{A})$ and obese $(n=6)$ subjects $(\mathbf{B})$. (C) Firmicutes/Bacteroidetes ratio of all subjects. Data are shown as mean \pm standard error of the mean (SEM). $(A, C)$ Significant differences are indicated as ${ }^{*}$ adj. $p$-value $<0.05$. (B) Significant differences are indicated as * $p$-value $<0.05$.

\subsection{Fructose Dependent Changes of Relative Abundance on Phylum Level}

Analyzing bacterial abundances on the genus level, we identified ten genera that were significantly different among the diet phases, Parabacteroides, Alistipes, Odoribacter, Oscillibacter, Faecalibacterium, Barnesiella, Erysipelatoclostridium, Ruminococcus, Anaerostipses and Veillonella if no correction for multiple testing was applied. Abundance of the genus Parabacteroides was significantly reduced after the fruit diet compared to the low-fructose diet (lowf1), and abundance of Anaerostipes was significantly increased (Figure 4A). Alistipes, Oscillibacter, Odoribacter and Barnesiella were significantly decreased after the fruit diet compared to the low-fructose diet (lowf2) (Figure 4B). Abundance of the two genera Faecalibacterium and Erysipelatoclostridium was increased during the fruit diet and significantly decreased between the fruit and the lowf2 phase (Figure 4B). Comparing abundances between the lowf2 and HFS diet, Ruminococcus was reduced by the high-fructose syrup diet ( $p=0.052$, Figure $4 \mathrm{C})$. Abundances differed also among the two high-fructose diets, the fruit diet, which is characterized by a high fiber intake, and the HFS diet. The relative abundances of Ruminococcus and Erysipelatoclostridium were significantly lower after the HFS diet compared to the fruit diet whereas Barnesiella abundance was significantly higher (Figure $4 \mathrm{E}$ ). When comparing abundances between the HFS diet and the lowf1 diet, we found that the abundance of the genus Veillonella was significantly higher after the high-fructose diet (data not shown), although the genus was in general of low abundance $(<1 \%)$. Abundance of Parabacteroides significantly decreased after the HFS diet compared to the lowf1 diet 
(Figure 4D). Microbiota profiles of lean and obese subjects showed similar patterns for most genera during the study phases though these changes were not significant (data not shown). Ruminococcus was the only genus the abundance of which was significantly reduced by the high-fructose syrup in the lean subject group but not in the obese subject group (data not shown).

A

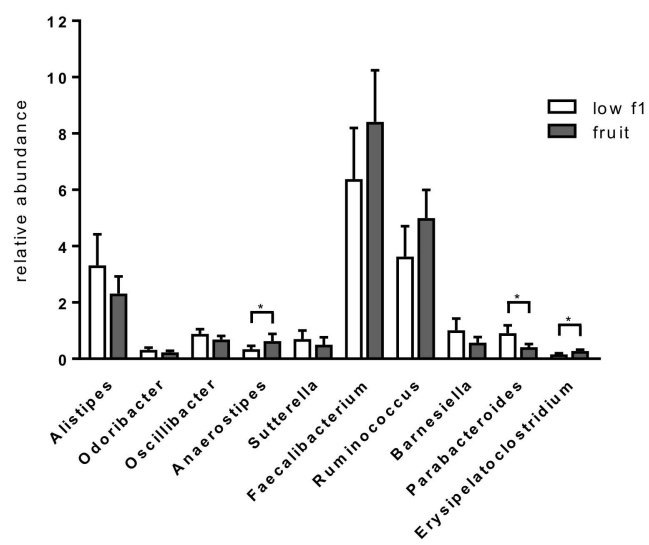

C

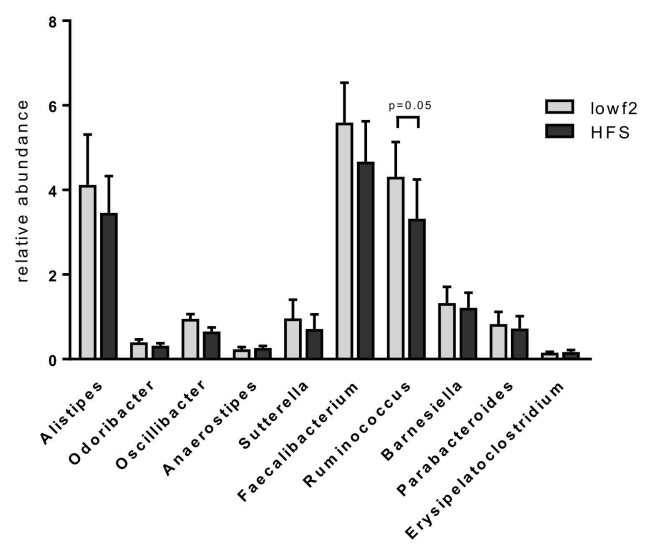

E

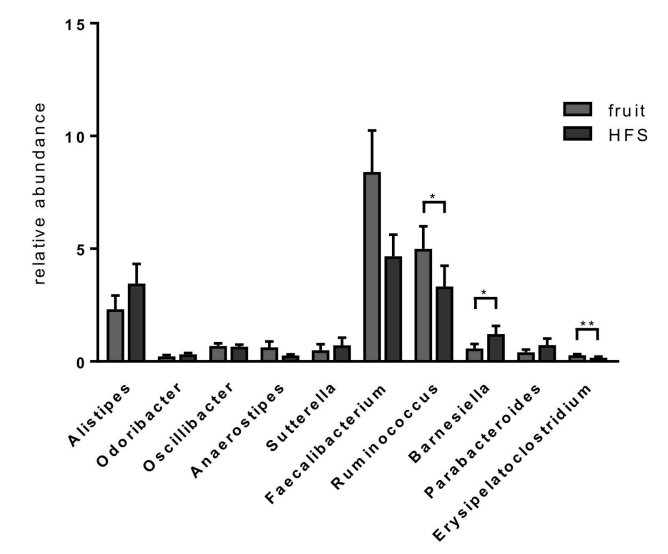

B

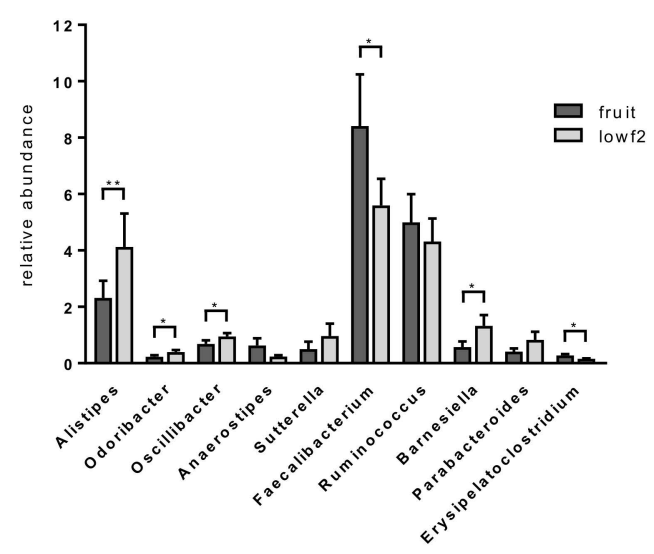

D

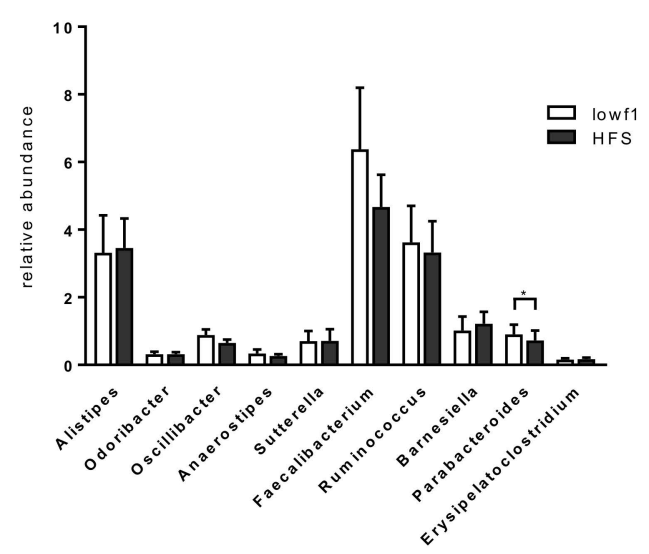

Figure 4. Changes of gut microbiota (relative abundances) on genus level among different fructose diets. (A) lowf1 vs. fruit, (B) fruit vs. lowf2, (C) lowf2 versus high-fructose syrup (HFS), (D) lowf1 vs. HFS, (E) fruit versus HFS. Significant differences are indicated as ${ }^{*} p$-value $<0.05$ and ${ }^{* *} p$-value $<0.01$. 


\subsection{Functional Alterations in the Gut Microbiome during the Response to Fructose}

The Kyoto Encyclopedia of Genes and Genomes (KEGG) database analysis revealed a total of 18 biological metabolic pathways involved in the response to fructose, including essential carbohydrate metabolic pathway and amino acid metabolic pathways, fructose and mannose metabolism (K15856), and ascorbate and aldarate metabolism (K13874) (Table S1). The fructose and mannose metabolism pathway were significantly elevated after the fruit diet (adjusted $p=0.011$, Table S1). The fruit diet also significantly changed the bacterial chemotaxis pathway, the folate biosynthesis pathway and the ABC transporter pathway. In particular, GDP-4-dehydro-6-deoxy-D-mannose reductase (K15856), chemotaxis protein CheX (K03409), molybdenum cofactor cytidylyltransferase (K07141), putative lysine transport system substrate-binding protein (K17073) were all significantly higher after the fruit diet. When analyzing changes between lowf2 and HFS diet, three pathways were affected, the inositol phosphate metabolism, the ascorbate and aldarate metabolism and the two-component system. We found scyllo-inositol 2-dehydrogenase (NAD +) (K16043) significantly more abundant after the HFS diet (adjusted $p<0.05$, Table S1) and LysR family transcriptional regulator (K18900) and L-arabinonolactonase (K13874) significantly reduced after the HFS diet (adjusted $p<0.05$, Table S1). The strongest effect of HFS was observed on the inositol phosphate metabolism (adjusted $p=6.15 \times 10^{-22}$, Table S1). Comparing the fruit diet with the HFS diet, 14 pathways were significantly changed, including the two-component system, fructose and mannose metabolism, glycerolipid metabolism, pyrimidine metabolism, purine metabolism, oxidative phosphorylation, folate biosynthesis, flagellar assembly, bacterial chemotaxis. From the fructose and mannose metabolism the GDP-4-dehydro-6-deoxy-D-mannose reductase (K15856) significantly decreased (adjusted $p=0.046$, Table S1).

\subsection{Correlation Analysis between Microbiota Abundance and Clinical Parameters.}

To evaluate the association between the clinical parameters and bacterial taxa, a correlation analysis was performed with abundances of all bacterial phyla, families and genera of the study subjects during the four different dietary phases. Correlations within all bacterial taxon and clinical parameters are shown in the supplemental material (Figure S1). Abundance of Bacteroidetes positively correlated with plasma cholesterol $(r=0.407, p=0.004)$ and LDL level $(r=0.362, p=0.012)$ (Figure 5A,B). In contrast, abundance of Firmicutes was negatively correlated with cholesterol $(r=-0.350, p=0.016)$ and LDL level ( $r=-0.292, p=0.047$ (Figure 5C,D). On the genus level, abundance of Parabacteroides was positively correlated with cholesterol $(r=0.544, p<0.001)$ and LDL level $(r=0.449, p=0.002)$ (Figure 5E,F). Abundance of Sutterella was positively correlated with plasma LDL $(r=0.390, p=0.007)$, TG $(r=0.574, p<0.001)$ and cholesterol level $(r=0.432, p=0.002)$ (Figure S1). Plasma levels of LDL were also correlated with the abundance of Alistipes $(r=-0.591, p<0.001)$ (Figure 5G). Furthermore, a positive correlation between abundance of Ruminococcus and levels of ALT was observed $(r=0.383$, $p=0.008$ ) (Figure 5H). 
A

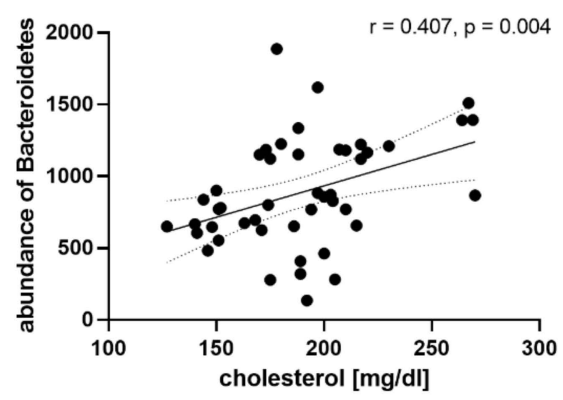

C

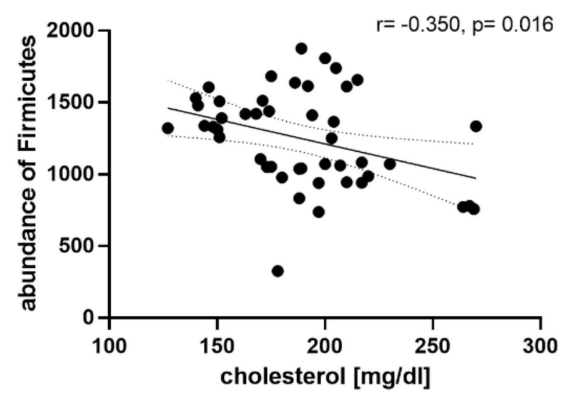

$\mathbf{E}$

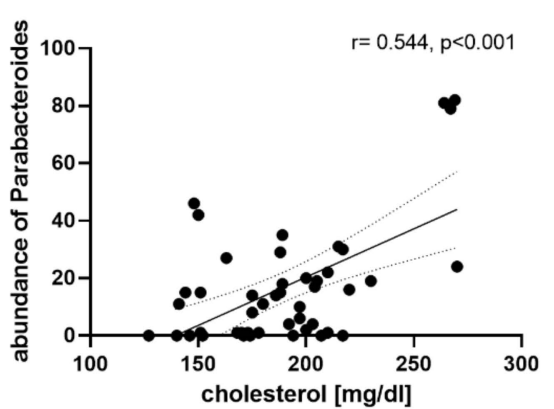

G

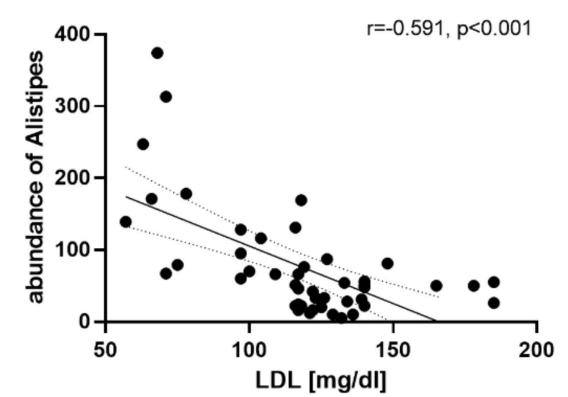

B

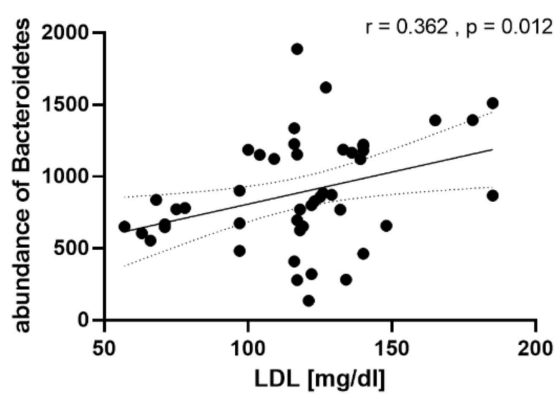

D

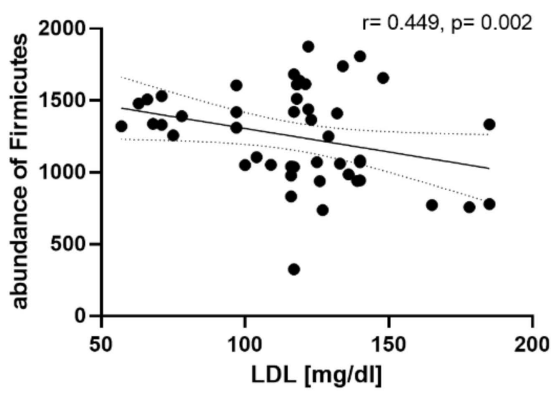

$\mathbf{F}$

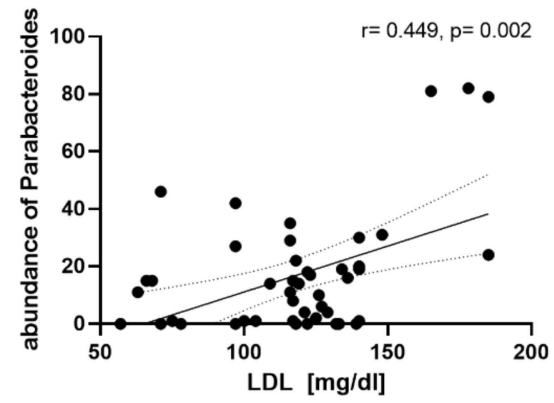

H

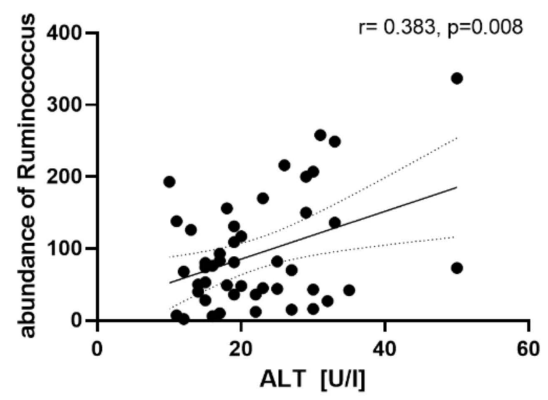

Figure 5. Abundances of specific phyla and genera that correlate with plasma lipid parameters. Bacteroidetes abundance vs. cholesterol (A) and LDL level (B), Firmicutes abundance vs. cholesterol (C) and LDL level (D), Parabacteroides abundance vs. cholesterol (E) and LDL level (F). Alistipes abundance vs. LDL level (G), Ruminococcus abundance vs. ALT level (H). Pearson's correlation analysis is shown. Each dot represents a related pair of values from the study subjects. LDL, low density lipoprotein; ALT, alanine aminotransferase. 


\subsection{Correlation Analysis between Microbiota Abundance and Plasma Metabolites}

To focus our analysis further, we correlated the abundance of the genera with plasma metabolites acylcarnitines (AC), lysophosphatidylcholines (lysoPC), diacyl-phosphatidylcholines (PCaa) and acyl-phosphatidylcholines (PCae). The most abundant genus Faecalibacterium of the Firmicutes correlated positively with two long-chain ACs, whereas the second most abundant one, Ruminococcus, showed a positive correlation with short-chain ACs, specifically with AC C4 (Butyryl-L-carnitine) among others (Figure 6A-D). Within the Bacteroidetes taxon, mostly negative correlations to ACs were observed for Bacteroides (e.g., C3) and Barnesiella (e.g., C4) and positive ones for Alistipes and Prevotella (e.g., C2, Acetyl-L-carnitine). Parasutterella abundance (Proteobacteria) was negatively correlated with ACs whereas Bifidobacteria (Actinobacter) showed a positive correlation to C9 (Figure 6A). A few long-chain lysoPCs correlated in both directions with the abundance of Ruminococcus. Within the Bacteroidetes taxon, prevailing positive (Alistipes and Prevotella) but also negative (Barnesiella) correlations were found. Parasutterella (Proteobacteria) correlated negatively to some lysoPCs (Figure 6B). Within the Firmicutes taxon, PCaas correlated in both directions largely equilibrated whereby Bacteroides (Bacteroidetes) were found to correlate mostly positively (Figure 6C). Bacteria from the Firmicutes and Bacteroidetes taxon were found to correlate with PCaes approximately equilibrated in both directions (Figure 6D). 

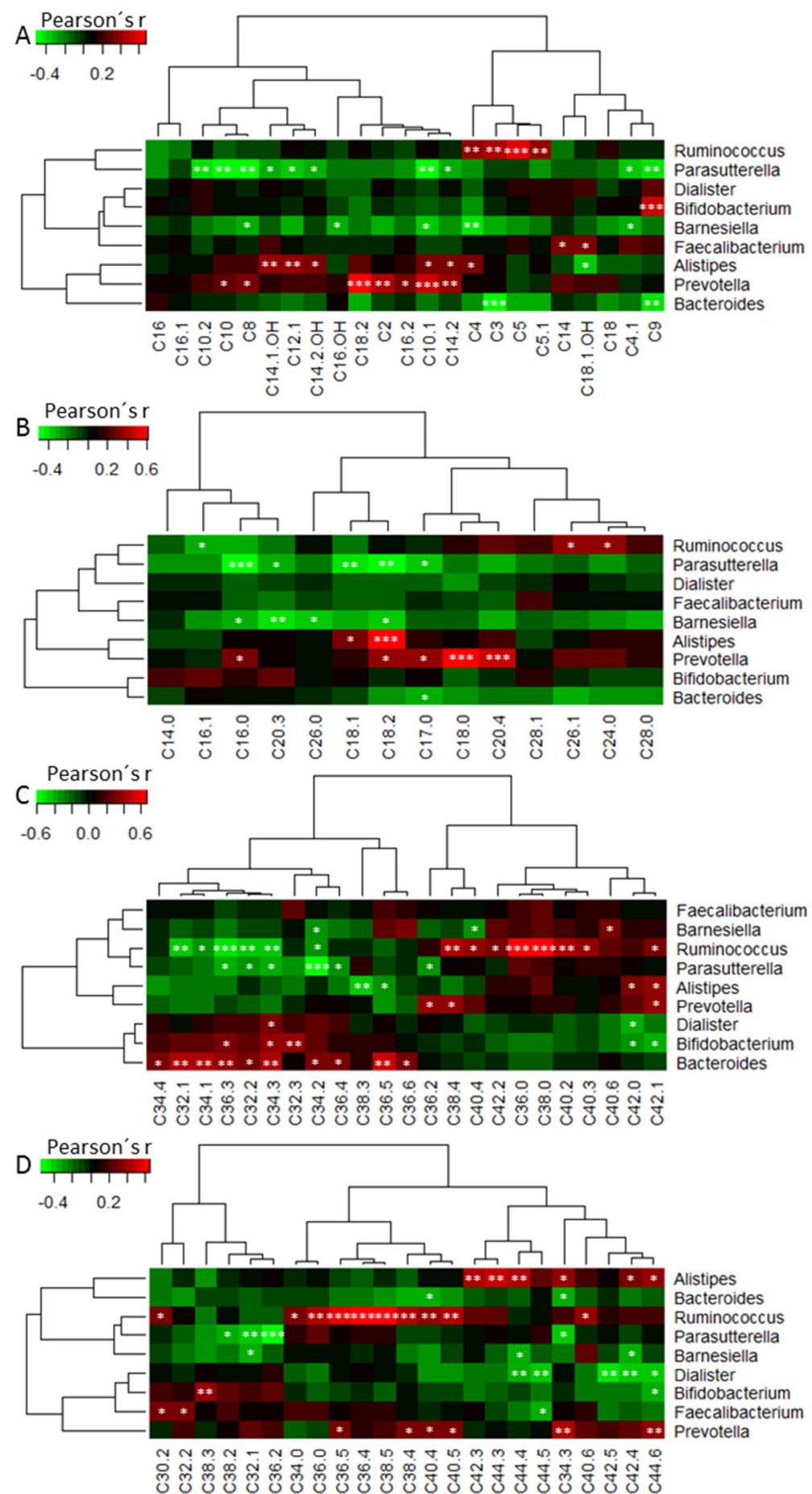

Figure 6. Correlation heatmap (Pearson's correlation) of most abundant gut microbiota on genus level and plasma metabolites. Microbiota vs. (A) acylcarnitines, (B) lyso-phosphatidylcholines, (C) diacyl-phosphatidylcholines, (D) acyl-phosphatidylcholines. Green colored field figure negative and red colored positive correlations. Hierarchical clustering of microbiota abundance and clinical parameter are shown by dendograms.

\section{Discussion}

In the present study, we demonstrate that diets differing in the amount of the fructose content and the source of the fructose mediate alterations of the gut microbiota differently in healthy humans. Abundance of the phyla Firmicutes and Bacteroidetes, which represent the largest proportion of the gut microbiota, changed in response to the different high-fructose diets. The relative abundance 
of Firmicutes increased after a fruit diet and decreased following the HFS diet, whereas the relative abundance of Bacteroidetes showed opposing patterns. At the genus level, we observed more specific shifts including an increase in Faecalibacterium and Anareostipes and a reduction in Parabacteroides and Barnesiella after the fruit-rich diet. After the high-fructose syrup diet (HFS), we observed a decrease of Ruminococcus, Faecalibacterium and Erysipelatoclostridium whereas Barnesiella abundance was higher after the HFS diet.

High consumption of fructose, one of the critical risk factors contributing to the development of NAFLD, increases gut permeability and alters the microbiota composition in the gastrointestinal tract and therefore promotes bacterial translocation and metabolic endotoxemia resulting in lipid accumulation and low-grade inflammation leading finally to hepatic steatosis and metabolic disease. Low doses of fructose are cleared by the small intestine, but high doses of fructose saturate the absorption and catabolism of fructose leading to fructose spill-over not only to the liver [42] but also to the colonic microbiota, which further metabolizes fructose [43]. The resulting metabolites become an energy source for bacteria and influence the gut environment [44]. Here we report, for the first time, that the human intestinal microbiota is altered differently in response to dietary fructose in different concentrations and sources, which is uncovered by significant taxonomic differences between the different fructose diet phases.

Our finding of an increased Firmicutes and a reduced Bacteroidetes abundance in healthy adults after a high-fructose diet derived from fruit and vegetables confirms and extents previous findings. The first evidence that fructose might affect microbiota arose from studies in rodent models [28,45]. Fructose-fed mice showed a significantly lower abundance of Bacteroidetes and a slightly increased Firmicutes abundance [26]. The microbiota changes we observed at the phylum level are consistent with these data and also in agreement with our own previous findings that a high-fructose diet caused a reduced Bacteroidetes abundance and a slightly enhanced Firmicutes abundance [6]. Furthermore, our data are in accordance with a study by Astbury et al. [46], who found a higher Firmicutes and a lower Bacteroides abundance after a high-fructose diet during pregnancy in rats. However, it should be noted that all these studies analyzed the effects of fructose either added to the solid diet or as an additive in drinking water, but not as fructose from whole fruits. As a result of a lower Bacteroidetes and a higher Firmicutes abundance, the Firmicutes to Bacteroidetes $(F / B)$ ratio was slightly increased after the fruit diet. This is in contrast to previous literature which proposed the $F / B$ ratio to be a contributing factor to obesity and its relative metabolic disease although results in humans are controversial. Several studies did not observe any changes in the $F / B$ ratio or even reported a decreased ratio in obese human individuals [47-49]. Others reported an increased F/B ratio only in obese with metabolic alterations [50]. The discrepancies may relate to methodological differences between studies, to population differences (obese individuals with or without metabolic disease) or to dietary influences.

Besides a high fructose content, the fruit diet was characterized by a relatively high content of fiber, and study subjects had a significantly higher fiber intake during the fruit phase than during the HFS diet. The observed alterations in gut microbiota may at least partially reflect changes driven by a higher intake of fiber rather than changes in response to the high-fructose consumption. Our results are in line with the results of Wu et al. who reported that abundance of Firmicutes was positively associated with a high-fiber diet, whereas Bacteroidetes abundance was predominantly negatively associated with fiber intake [51]. Firmicutes abundance was also increased in humans following a short-term dietary intervention characterized by a high-fiber content, and this was accompanied by a decreased abundance of several genera from the Bacteroidetes phylum [52]. Similarly, other studies described a decreased abundance of the Bacteroidetes taxon after a fiber rich diet, e.g., of the class Bacteroidia [53] and genus Bacteroides [54]. Consistent with these data, we observed a lower abundance of Bacteroidetes after the fruit diet. Abundance of Barnesiella, a common gut bacterium present in healthy individuals and genus of the order Bacteroidales, was also significantly reduced after the fruit diet compared to lowf2 diet phase. 
Dietary fibers from fruit and vegetable are degraded by colonic bacteria resulting in the production of key metabolites such as short chain fatty acids (SCFAs), which are important promoters of gut health. SCFAs, particularly butyrate, are well known for their anti-inflammatory functions [55], antimicrobial activity and maintenance of intestinal barrier function and may be protective against several diseases, including diabetes and obesity [56]. Interestingly, in our study, a high intake of fructose from fruit and vegetables increased abundance of the genera Anaerostipes, Coprococcus, Ruminococcus (though effects for Coprococcus and Ruminococcus were not significant) and Erysipelatoclostridium all belonging to butyrate-producing bacteria of the phylum Firmicutes. The two families Lachnospiraceae and Ruminococcacae include most of the known butyrate producers $[57,58]$. The significantly increased abundance of Anaerostipes (family Lachnospiraceae) after the fruit diet is supported by previous findings describing the ability to metabolize fructose [59,60]. Furthermore, our results are in line with a previous study showing that a fructose-supplemented diet increased the abundance of Anaerostipes genus [61]. The high-fructose fruit diet also enriched abundance of Coprococcus (family Lachnospiraceae) and Ruminococcus (family Ruminococcacae). Interestingly, the abundance of Ruminococcus was positively associated with short-chain ACs, among others with AC C4 (Butyryl-L-carnitine) suggesting that butyrate delivered by Ruminococcus might be responsible for the elevated blood AC C4:1 level after the fruit diet. Similar changes in the gut microbiota have been observed in studies in which the abundance of the genera Coprococcus and Ruminococcus was increased by a fructose-rich diet in mice $[28,29,62,63]$. One of the most abundant butyrate producers in the human gut is Faecalibacterium prausnitzi, which also ferments fructose [64]. Supporting the hypothesis that a fructose and fiber-rich diet shifts microbiota towards butyrate producers we found the genus Faecalibacterium significantly increased after the fruit diet compared to the low-fructose diet. In summary, a high-fructose fruit diet led to an increase of butyrate-producing bacteria, which may promote a healthy gut function.

A clear increase in the consumption of fructose has occurred in recent decades particularly in form of HFS, which is used as sweetener in soft drinks and other sweetened beverages and in processed foods [12]. Western-style diets high in HFS not only increase the risk of NAFLD but also contribute to the rise and prevalence of obesity [65]. Most recently, the demonstration that dietary fructose stimulates hepatic lipogenesis via microbiota-derived acetate determined a previously unappreciated interaction among diet, the gut microbiome and the host metabolism that contributes to fructose-induced NAFLD [66]. In the current study, we found that the gut microbiota composition was distinctively different after a short-term HFS diet compared to after the fruit-rich diet. This difference might be related to different absorption rates for fructose in the small intestine depending on the fruit matrix or the fiber content leading to different outcomes in microbiota metabolism. The observation of an increased abundance of Bacteroidetes after the HFS diet aligns with a previously reported increase in Bacteroides after high-fructose syrup liquid diet in mice [62] and an increased abundance of Bacteroidetes in rats fed a high-fructose diet [30]. Bacteroidetes bacteria, which compromise the largest phylum of Gram-negative bacteria in the gut microbiome, are the most abundant contributors to LPS biosynthesis [67]. A high abundance of Bacteroidetes has been associated with liver inflammation $[25,68,69]$ and the development of NAFLD and further metabolic diseases [70]. The correlation between the Bacteroidetes abundance and blood cholesterol and LDL levels supports the hypothesis that a higher Bacteroidetes abundance induced by the HFS diet may be associated with increased blood lipid levels. A decreased abundance of Firmicutes after the HFS diet, which was negatively correlated with blood cholesterol and LDL levels, matches these findings and further supports the assumption that HFS diet-induced microbiota changes may have unfavorable health effects.

Interestingly, within the phylum Firmicutes, abundance of Erysipelatoclostridium and Ruminococcus was reduced after the HFS diet compared to the fruit diet, whereas abundance of Veillonella was increased after the HFS compared to the lowf1 diet. Some Veillonella species are able to ferment fructose and fructose can be incorporated into the Veillonella LPS and thus may be of major importance in the production of LPS [71]. We therefore hypothesize that an increased Veillonella abundance induced by the HFS diet could contribute to the production of endotoxins and increase LPS level. Our results are 
in line with a recent study by Li et al. [72] who found the genus Veillonella to have increased after a high-fructose diet in mice.

In contrast to studies in rodent models reporting an increased Ruminococcus abundance after a high-fructose diet $[6,28,29]$, Ruminococcus abundance was not significantly changed following the HFS diet compared to low fructose diet. These differences might be at least partially explained by different diet formulations as in the animal experiments fructose was incorporated in the solid diet whereas our study analyzed the effect of high-fructose corn syrup. In addition, caution should be taken in interpreting studies of the fructose effects in animal experiments because results from animal models cannot always be translated to humans. Compared to the high-fructose fruit diet Ruminococcus was of significantly lower abundance after the HFS diet hinting at a dietary fiber-related effect. Analyzing the lean study group separately, we found Ruminococcus being significantly reduced by the HFS diet compared to the lowf2 diet. As study subjects had a similar intake of fiber and fat during the lowf2 and the HFS diet phases, this effect cannot be attributed to differences in the consumption of dietary fiber. Bacteria of the genus Ruminococcus are key players in the dynamics of gut microbial communities and have been associated with gastrointestinal health benefits in humans [73]. In summary, our results point to a reduction of the beneficial butyrate producing Ruminococcus bacteria after the HFS diet. Furthermore, our data suggest that the HFS syrup containing a mix of fructose and glucose as monosaccharides leads to a different outcome in microbiota metabolism compared to dietary fructose from fruit and vegetable where it is also found bonded to glucose as the disaccharide sucrose and thus affects microbiota composition and Ruminococcus abundance differently. Besides this, intake of dietary glucose during the HFS diet was significantly higher than during the fruit diet (data not shown), which could also play a role in different effects of the two high-fructose diets. Furthermore, fructose from HFS in contrast to fructose from fruit and vegetables, which is absorbed and released consistently from the food matrix in smaller amounts, may persist for a longer time within the gut and, thus, be metabolized by enterocytes and potentially by microbiota of the large intestine.

Remarkably, abundance of Parabacteroides, known for its anti-obesity effects, was significantly decreased by both high-fructose diets compared to the lowf1 diet though the HFS diet-induced decrease was less pronounced. These results are consistent with findings from our own group demonstrating that Parabacteroides abundance was reduced after a high-fructose diet [6]. Our observations align with a previous report in which Parabacteroides species were exclusively observed in rats fed a control diet and not in a fructose-rich diet [46]. Recently, the gut commensal Parabacteroides distasonis was shown to alleviate obesity [74] further strengthening the assumption that a high-fructose diet hampers health promoting microbiota and might thus promote metabolic diseases.

A potential limitation of this study is the small sample size, which, together with a high inter-individual variability, may have affected the statistical power. Differences in the baseline microbial composition can drive the response to dietary changes $[75,76]$, which is highly individualized [77,78]. Interindividual differences in the response to dietary fructose might therefore have weakened mean microbiota changes between different diet groups. Furthermore, one-week dietary phases might have led us to miss potential long-term effects on microbiota. Though rearrangements in the human microbiome have been observed $24 \mathrm{~h}$ after initiating a diet $[47,79]$, most of the studies analyzed short-term effects on microbiota composition after two weeks of dietary intervention. While a significant decrease of gut community diversity was found after two weeks of short-term diet [48], no significant differences in $\alpha$-diversity were detected after a short-term diet of 5 days [80]. This is in line with our observation that $\alpha$-diversity did not change significantly between the one-week diet phases. Because of the short-term intervention phases, carry-over effects, namely, during the low f2 phase, cannot be excluded. This might explain some differences we found between the low f1 and low f2 phases despite of the fact that the diet was the same in these two phases. 


\section{Conclusions}

In conclusion, our results demonstrate that short-term high-fructose diets differing in both amount and source of fructose mediate alterations of the gut microbiota composition differently. Importantly, we provide evidence that the HFS diet induces an imbalanced microbiota profile characterized by a significantly reduced abundance of beneficial butyrate-producing bacteria and of bacteria known for anti-obesity effects which could cause disruption of mucosal homeostasis and gut function.

Despite the high-fructose content, the fruit-rich diet shifts the intestinal microbiota composition towards a healthy butyrate-producing community, suggesting that effects which may be attributed to fiber content of fruit and vegetable prevail and influence the microbiota composition in an protective manner counteracting the harmful effects of excessive fructose. However, future studies are needed to further investigate the mechanism of action by which fructose elicits its versatile functions on host microbiota and the role of fructose-mediated changes in the gut microbiota.

Supplementary Materials: The following are available online at http://www.mdpi.com/2072-6643/12/11/3444/s1. Figure S1: Correlation heatmap (Pearson's correlation) of gut microbiota within 6 taxon levels and clinical parameters. Table S1: KEGG modules significantly associated with diet.

Author Contributions: A.D.-M. and S.C.B. designed the study. A.D.-M. and M.B. conducted the study and acquired data. J.B. and A.G.-G. analyzed the data and interpreted results. J.B. and A.G.-G. prepared figures. J.B. and A.G.-G. wrote the manuscript. A.D.-M., M.B. and S.C.B. approved final version of manuscript. All authors have read and agreed to the published version of the manuscript.

Funding: This work was supported in part with grants from the Competence Network of Obesity, group "Obesity and GI tract", funded by the Federal Ministry of Education and Research (FKZ: 01GI0843). The funders had no role in study design, data collection and analysis, decision to publish or preparation of the manuscript.

Acknowledgments: Authors would like to thank Adelbert Roscher for scientific advice and support.

Conflicts of Interest: The authors declare no conflict of interest.

\section{References}

1. World Health Organization. Fact-Sheet on Obesity and Overweight. Available online: http://www.who.int/ news-room/fact-sheets/detail/obesity-and-overweight (accessed on 22 December 2018).

2. White, J.S. Challenging the Fructose Hypothesis: New Perspectives on Fructose Consumption and Metabolism. Adv. Nutr. 2013, 4, 246-256. [CrossRef] [PubMed]

3. Kavanagh, K.; Wylie, A.T.; Tucker, K.L.; Hamp, T.J.; Gharaibeh, R.Z.; Fodor, A.A.; Cullen, J.M.C. Dietary fructose induces endotoxemia and hepatic injury in calorically controlled primates. Am. J. Clin. Nutr. 2013, 98, 349-357. [CrossRef]

4. Crescenzo, R.; Bianco, F.; Falcone, I.; Coppola, P.; Liverini, G.; Iossa, S. Increased hepatic de novo lipogenesis and mitochondrial efficiency in a model of obesity induced by diets rich in fructose. Eur. J. Nutr. 2013, 52, 537-545. [CrossRef] [PubMed]

5. Volynets, V.; Louis, S.; Pretz, D.; Lang, L.; Ostaff, M.J.; Wehkamp, J.; Bischoff, S.C. Intestinal Barrier Function and the Gut Microbiome Are Differentially Affected in Mice Fed a Western-Style Diet or Drinking Water Supplemented with Fructose. J. Nutr. 2017, 147, 770-780. [CrossRef] [PubMed]

6. Crescenzo, R.; Cigliano, L.; Mazzoli, A.; Cancelliere, R.; Carotenuto, R.; Tussellino, M.; Liverini, G.; Iossa, S. Early Effects of a Low Fat, Fructose-Rich Diet on Liver Metabolism, Insulin Signaling, and Oxidative Stress in Young and Adult Rats. Front. Physiol. 2018, 9, 411. [CrossRef] [PubMed]

7. Aeberli, I.; Gerber, P.A.; Hochuli, M.; Kohler, S.; Haile, S.R.; Gouni-Berthold, I.; Berthold, H.K.; Spinas, G.A.; Berneis, K. Low to moderate sugar-sweetened beverage consumption impairs glucose and lipid metabolism and promotes inflammation in healthy young men: A randomized controlled trial. Am. J. Clin. Nutr. 2011, 94, 479-485. [CrossRef]

8. Stanhope, K.L.; Schwarz, J.M.; Keim, N.L.; Griffen, S.C.; Bremer, A.A.; Graham, J.L.; Hatcher, B.; Cox, C.L.; Dyachenko, A.; Zhang, W.; et al. Consuming fructose-sweetened, not glucose-sweetened, beverages increases visceral adiposity and lipids and decreases insulin sensitivity in overweight/obese humans. J. Clin. Investig. 2009, 119, 1322-1334. [CrossRef] 
9. Stanhope, K.L.; Medici, V.; Bremer, A.A.; Lee, V.; Lam, H.D.; Nunez, M.V.; Chen, G.X.; Keim, N.L.; Havel, P.J. A dose-response study of consuming high-fructose corn syrup-sweetened beverages on lipid/lipoprotein risk factors for cardiovascular disease in young adults. Am. J. Clin. Nutr. 2015, 101, 1144-1154. [CrossRef]

10. Raatz, S.K.; Johnson, L.K.; Picklo, M.J. Consumption of Honey, Sucrose, and High-Fructose Corn Syrup Produces Similar Metabolic Effects in Glucose-Tolerant and -Intolerant Individuals. J. Nutr. 2015, 145, 2265-2272. [CrossRef]

11. Siqueira, J.; Mill, J.; Velasquez-Melendez, G.; Moreira, A.D.; Barreto, S.M.; Benseñor, I.M.; Molina, M.D.C.B. Sugar-Sweetened Soft Drinks and Fructose Consumption Are Associated with Hyperuricemia: Cross-Sectional Analysis from the Brazilian Longitudinal Study of Adult Health (ELSA-Brasil). Nutrients 2018, 10, 981. [CrossRef]

12. Bray, G.A.; Nielsen, S.J.; Popkin, B.M. Consumption of high-fructose corn syrup in beverages may play a role in the epidemic of obesity. Am. J. Clin. Nutr. 2004, 79, 537-543. [CrossRef] [PubMed]

13. Ferder, L.; Ferder, M.D.; Inserra, F. The Role of High-Fructose Corn Syrup in Metabolic Syndrome and Hypertension. Curr. Hypertens. Rep. 2010, 12, 105-112. [CrossRef] [PubMed]

14. Lambertz, J.; Weiskirchen, S.; Landert, S.; Weiskirchen, R. Fructose: A Dietary Sugar in Crosstalk with Microbiota Contributing to the Development and Progression of Non-Alcoholic Liver Disease. Front. Immunol. 2017, 8, 1159. [CrossRef] [PubMed]

15. Ouyang, X.; Cirillo, P.; Sautin, Y.; McCall, S.; Bruchette, J.L.; Diehl, A.M.; Johnson, R.J.; Abdelmalek, M.F. Fructose consumption as a risk factor for non-alcoholic fatty liver disease. J. Hepatol. 2008, 48, 993-999. [CrossRef]

16. Thuy, S.; Ladurner, R.; Volynets, V.; Wagner, S.; Strahl, S.; Königsrainer, A.; Maier, K.-P.; Bischoff, S.C.; Bergheim, I. Nonalcoholic Fatty Liver Disease in Humans Is Associated with Increased Plasma Endotoxin and Plasminogen Activator Inhibitor 1 Concentrations and with Fructose Intake. J. Nutr. 2008, 138, 1452-1455. [CrossRef]

17. Volynets, V.; Machann, J.; Küper, M.A.; Maier, I.B.; Spruss, A.; Königsrainer, A.; Bischoff, S.C.; Bergheim, I. A moderate weight reduction through dietary intervention decreases hepatic fat content in patients with non-alcoholic fatty liver disease (NAFLD): A pilot study. Eur. J. Nutr. 2013, 52, 527-535. [CrossRef]

18. Rahman, K.; Desai, C.; Iyer, S.S.; Thorn, N.E.; Kumar, P.; Liu, Y.; Smith, T.; Neish, A.S.; Li, H.; Tan, S.; et al. Loss of Junctional Adhesion Molecule A Promotes Severe Steatohepatitis in Mice on a Diet High in Saturated Fat, Fructose, and Cholesterol. Gastroenterology 2016, 151, 733-746.e12. [CrossRef]

19. Kawabata, K.; Kanmura, S.; Morinaga, Y.; Tanaka, A.; Makino, T.; Fujita, T.; Arima, S.; Sasaki, F.; Nasu, Y.; Tanoue, S.; et al. A high-fructose diet induces epithelial barrier dysfunction and exacerbates the severity of dextran sulfate sodium-induced colitis. Int. J. Mol. Med. 2019, 43, 1487-1496. [CrossRef]

20. Ritze, Y.; Bárdos, G.; Hubert, A.; Böhle, M.; Bischoff, S.C. Effect of tryptophan supplementation on diet-induced non-alcoholic fatty liver disease in mice. Br. J. Nutr. 2014, 112, 1-7. [CrossRef]

21. Cani, P.D.; Bibiloni, R.; Knauf, C.; Waget, A.; Neyrinck, A.M.; Delzenne, N.M.; Burcelin, R. Changes in Gut Microbiota Control Metabolic Endotoxemia-Induced Inflammation in High-Fat Diet-Induced Obesity and Diabetes in Mice. Diabetes 2008, 57, 1470-1481. [CrossRef]

22. Farhadi, A.; Gundlapalli, S.; Shaikh, M.; Frantzides, C.; Harrell, L.; Kwasny, M.; Keshavarzian, A. Susceptibility to gut leakiness: A possible mechanism for endotoxaemia in non-alcoholic steatohepatitis. Liver Int. 2008, 28, 1026-1033. [CrossRef] [PubMed]

23. Scott, K.P.; Gratz, S.W.; Sheridan, P.O.; Flint, H.J.; Duncan, S.H. The influence of diet on the gut microbiota. Pharmacol. Res. 2013, 69, 52-60. [CrossRef] [PubMed]

24. Sen, T.; Cawthon, C.R.; Ihde, B.T.; Hajnal, A.; DiLorenzo, P.M.; De La Serre, C.B.; Czaja, K. Diet-driven microbiota dysbiosis is associated with vagal remodeling and obesity. Physiol. Behav. 2017, 173, 305-317. [CrossRef] [PubMed]

25. Ferrere, G.; Leroux, A.; Wrzosek, L.; Puchois, V.; Gaudin, F.; Ciocan, D.; Renoud, M.-L.; Naveau, S.; Perlemuter, G.; Cassard, A.-M. Activation of Kupffer Cells Is Associated with a Specific Dysbiosis Induced by Fructose or High Fat Diet in Mice. PLoS ONE 2016, 11, e0146177. [CrossRef]

26. Do, M.H.; Lee, E.; Oh, M.-J.; Kim, Y.; Park, H.-Y. High-Glucose or -Fructose Diet Cause Changes of the Gut Microbiota and Metabolic Disorders in Mice without Body Weight Change. Nutrients 2018, 10, 761. [CrossRef] 
27. Chen, Y.; Zhu, Y.; Wu, C.; Lu, A.; Deng, M.; Yu, H.; Huang, C.; Wang, W.; Li, C.; Zhu, Q.; et al. Gut dysbiosis contributes to high fructose-induced salt-sensitive hypertension in Sprague-Dawley rats. Nutrients 2020, 75-76, 110766. [CrossRef]

28. Di Luccia, B.; Crescenzo, R.; Mazzoli, A.; Cigliano, L.; Venditti, P.; Walser, J.-C.; Widmer, A.; Baccigalupi, L.; Ricca, E.; Iossa, S. Rescue of Fructose-Induced Metabolic Syndrome by Antibiotics or Faecal Transplantation in a Rat Model of Obesity. PLoS ONE 2015, 10, e0134893. [CrossRef]

29. Crescenzo, R.; Mazzoli, A.; Di Luccia, B.; Bianco, F.; Cancelliere, R.; Cigliano, L.; Liverini, G.; Baccigalupi, L.; Iossa, S. Dietary fructose causes defective insulin signalling and ceramide accumulation in the liver that can be reversed by gut microbiota modulation. Food Nutr. Res. 2017, 61, 1331657. [CrossRef]

30. Rivero-Gutierrez, B.; Gámez-Belmonte, R.; Suárez, M.D.; Olivares, M.; Martínez-Augustin, O.; Zarzuelo, A.; Lavín, J.L.; Aransay, A.M.; De Medina, F.S. A synbiotic composed of Lactobacillus fermentum CECT5716 and FOS prevents the development of fatty acid liver and glycemic alterations in rats fed a high fructose diet associated with changes in the microbiota. Mol. Nutr. Food Res. 2017, 61, 1600622. [CrossRef]

31. De-Bandt, J.-P.; Waligora-Dupriet, A.-J.; Butel, M.-J. Intestinal microbiota in inflammation and insulin resistance: Relevance to humans. Curr. Opin. Clin. Nutr. Metab. Care 2011, 14, 334-340. [CrossRef]

32. Gonzalez-Granda, A.; Damms-Machado, A.; Basrai, M.; Bischoff, S.C. Changes in Plasma Acylcarnitine and Lysophosphatidylcholine Levels Following a High-Fructose Diet: A Targeted Metabolomics Study in Healthy Women. Nutrients 2018, 10, 1254. [CrossRef] [PubMed]

33. Mishra, P.; Younossi, Z.M. Abdominal Ultrasound for Diagnosis of Nonalcoholic Fatty Liver Disease (NAFLD). Am. J. Gastroenterol. 2007, 102, 2716-2717. [CrossRef] [PubMed]

34. Webb, M.; Yeshua, H.; Zelber-Sagi, S.; Santo, E.; Brazowski, E.; Halpern, Z.; Oren, R. Diagnostic Value of a Computerized Hepatorenal Index for Sonographic Quantification of Liver Steatosis. Am. J. Roentgenol. 2009, 192, 909-914. [CrossRef] [PubMed]

35. Bedogni, G.; Bellentani, S.; Miglioli, L.; Masutti, F.; Passalacqua, M.; Castiglione, A.; Tiribelli, C. The Fatty Liver Index: A simple and accurate predictor of hepatic steatosis in the general population. BMC Gastroenterol. 2006, 6, 33. [CrossRef]

36. Klindworth, A.; Pruesse, E.; Schweer, T.; Peplies, J.; Quast, C.; Horn, M.; Glöckner, F.O. Evaluation of general $16 \mathrm{~S}$ ribosomal RNA gene PCR primers for classical and next-generation sequencing-based diversity studies. Nucleic Acids Res. 2013, 41, e1. [CrossRef]

37. Schmieder, R.; Edwards, R. Quality control and preprocessing of metagenomic datasets. Bioinformatics 2011, 27, 863-864. [CrossRef]

38. Andrews, S. FastQC: A Quality Control Tool for High Throughput sequence Data. 2010. Available online: http://www.bioinformatics.babraham.ac.uk/projects/fastqc (accessed on 22 December 2018).

39. Aronesty, E. Comparison of Sequencing Utility Programs. Open Bioinform. J. 2013, 7, 1-8. [CrossRef]

40. Herbig, A.; Maixner, F.; Bos, K.I.; Zink, A.; Krause, J.; Huson, D.H. MALT: Fast alignment and analysis of metagenomic DNA sequence data applied to the Tyrolean Iceman. BioRxiv 2016, 050559. [CrossRef]

41. Huson, D.H.; Weber, N. Microbial community analysis using MEGAN. Methods Enzymol. 2013, 531, 465-485. [CrossRef]

42. Merino, B.; Fernández-Díaz, C.M.; Cózar-Castellano, I.; Perdomo, G. Intestinal Fructose and Glucose Metabolism in Health and Disease. Nutrients 2019, 12, 94. [CrossRef]

43. Jang, C.; Hui, S.; Lu, W.; Cowan, A.J.; Morscher, R.J.; Lee, G.; Liu, W.; Tesz, G.J.; Birnbaum, M.J.; Rabinowitz, J.D. The Small Intestine Converts Dietary Fructose into Glucose and Organic Acids. Cell Metab. 2018, 27, 351-361.e3. [CrossRef] [PubMed]

44. Ríos-Covián, D.; Ruas-Madiedo, P.; Margolles, A.; Gueimonde, M.; de Los Reyes-Gavilán, C.G.; Salazar, N. Intestinal Short Chain Fatty Acids and their Link with Diet and Human Health. Front. Microbiol. 2016, 7, 185. [CrossRef]

45. Jena, P.K.; Singh, S.; Prajapati, B.; Nareshkumar, G.; Mehta, T.; Seshadri, S. Impact of Targeted Specific Antibiotic Delivery for Gut Microbiota Modulation on High-Fructose-Fed Rats. Appl. Biochem. Biotechnol. 2014, 172, 3810-3826. [CrossRef] [PubMed]

46. Astbury, S.; Song, A.; Zhou, M.; Nielsen, B.; Hoedl, A.; Willing, B.P.; Symonds, M.E.; Bell, R.C. High Fructose Intake During Pregnancy in Rats Influences the Maternal Microbiome and Gut Development in the Offspring. Front. Genet. 2018, 9, 203. [CrossRef] 
47. Schwiertz, A.; Taras, D.; Schaefer, K.; Beijer, S.; Bos, N.A.; Donus, C.; Hardt, P.D. Microbiota and SCFA in Lean and Overweight Healthy Subjects. Obesity 2010, 18, 190-195. [CrossRef]

48. Duncan, S.H.; Lobley, G.E.; Holtrop, G.; Ince, J.; Johnstone, A.M.; Louis, P.; Flint, H.J. Human colonic microbiota associated with diet, obesity and weight loss. Int. J. Obes. (Lond.) 2008, 32, 1720-1724. [CrossRef] [PubMed]

49. Patil, D.P.; Dhotre, D.P.; Chavan, S.G.; Sultan, A.; Jain, D.S.; Lanjekar, V.B.; Gangawani, J.; Shah, P.S.; Todkar, J.S.; Shah, S.; et al. Molecular analysis of gut microbiota in obesity among Indian individuals. J. Biosci. 2012, 37, 647-657. [CrossRef] [PubMed]

50. Louis, S.; Tappu, R.-M.; Damms-Machado, A.; Huson, D.H.; Bischoff, S.C. Characterization of the Gut Microbial Community of Obese Patients Following a Weight-Loss Intervention Using Whole Metagenome Shotgun Sequencing. PLoS ONE 2016, 11, e0149564. [CrossRef]

51. Wu, G.D.; Chen, J.; Hoffmann, C.; Bittinger, K.; Chen, Y.-Y.; Keilbaugh, S.A.; Bewtra, M.; Knights, D.; Walters, W.A.; Knight, R.; et al. Linking Long-Term Dietary Patterns with Gut Microbial Enterotypes. Science 2011, 334, 105-108. [CrossRef]

52. Klimenko, N.S.; Tyakht, A.V.; Popenko, A.S.; Vasiliev, A.S.; Altukhov, I.A.; Ischenko, D.S.; Shashkova, T.I.; Efimova, D.A.; Nikogosov, D.A.; Osipenko, D.A.; et al. Microbiome Responses to an Uncontrolled Short-Term Diet Intervention in the Frame of the Citizen Science Project. Nutrients 2018, 10, 576. [CrossRef]

53. Lin, D.; Peters, B.A.; Friedlander, C.; Freiman, H.J.; Goedert, J.J.; Sinha, R.; Miller, G.; Bernstein, M.A.; Hayes, R.B.; Ahn, J. Association of dietary fibre intake and gut microbiota in adults. Br. J. Nutr. 2018, 120, 1014-1022. [CrossRef] [PubMed]

54. Hald, S.; Schioldan, A.G.; Moore, M.E.; Dige, A.; Lærke, H.N.; Agnholt, J.; Knudsen, K.E.B.; Hermansen, K.; Marco, M.L.; Gregersen, S.; et al. Effects of Arabinoxylan and Resistant Starch on Intestinal Microbiota and Short-Chain Fatty Acids in Subjects with Metabolic Syndrome: A Randomised Crossover Study. PLoS ONE 2016, 11, e0159223. [CrossRef] [PubMed]

55. Kim, C.H.; Park, J.; Kim, M. Gut Microbiota-Derived Short-Chain Fatty Acids, T Cells, and Inflammation. Immune Netw. 2014, 14, 277-288. [CrossRef] [PubMed]

56. Tan, J.; McKenzie, C.; Potamitis, M.; Thorburn, A.N.; Mackay, C.R.; Macia, L. The Role of Short-Chain Fatty Acids in Health and Disease. Adv. Immunol. 2014, 121, 91-119. [CrossRef]

57. Barcenilla, A.; Pryde, S.E.; Martin, J.C.; Duncan, S.H.; Stewart, C.S.; Henderson, C.; Flint, H.J. Phylogenetic Relationships of Butyrate-Producing Bacteria from the Human Gut. Appl. Environ. Microbiol. 2000, 66, 1654-1661. [CrossRef]

58. Louis, P.; Young, P.; Holtrop, G.; Flint, H.J. Diversity of human colonic butyrate-producing bacteria revealed by analysis of the butyryl-CoA:acetate CoA-transferase gene. Environ. Microbiol. 2010, 12, 304-314. [CrossRef]

59. Schwiertz, A.; Hold, G.L.; Duncan, S.H.; Gruhl, B.; Collins, M.D.; Lawson, P.A.; Flint, H.J.; Blaut, M. Anaerostipes caccae gen. nov., sp. nov., a New Saccharolytic, Acetate-utilising, Butyrate-producing Bacterium from Human Faeces. Syst. Appl. Microbiol. 2002, 25, 46-51. [CrossRef]

60. Allen-Vercoe, E.; Daigneault, M.; White, A.; Panaccione, R.; Duncan, S.H.; Flint, H.J.; O’Neal, L.; Lawson, P.A. Anaerostipes hadrus comb. nov., a dominant species within the human colonic microbiota; reclassification of Eubacterium hadrum Moore et al. 1976. Anaerobe 2012, 18, 523-529. [CrossRef]

61. Cho, S.; Tripathi, A.; Chlipala, G.; Green, S.; Lee, H.; Chang, E.B.; Jeong, H. Fructose diet alleviates acetaminophen-induced hepatotoxicity in mice. PLoS ONE 2017, 12, e0182977. [CrossRef]

62. Mastrocola, R.; Ferrocino, I.; Liberto, E.; Chiazza, F.; Cento, A.S.; Collotta, D.; Querio, G.; Nigro, D.; Bitonto, V.; Cutrin, J.C.; et al. Fructose liquid and solid formulations differently affect gut integrity, microbiota composition and related liver toxicity: A comparative in vivo study. J. Nutr. Biochem. 2018, 55, 185-199. [CrossRef]

63. Tain, Y.-L.; Lee, W.-C.; Wu, K.L.H.; Leu, S.; Chan, J.Y.H. Resveratrol Prevents the Development of Hypertension Programmed by Maternal Plus Post-Weaning High-Fructose Consumption through Modulation of Oxidative Stress, Nutrient-Sensing Signals, and Gut Microbiota. Mol. Nutr. Food Res. 2018, 62, e1800066. [CrossRef] [PubMed]

64. Duncan, S.H.; Hold, G.L.; Harmsen, H.J.M.; Stewart, C.S.; Flint, H.J. Growth requirements and fermentation products of Fusobacterium prausnitzii, and a proposal to reclassify it as Faecalibacterium prausnitzii gen. nov., comb. nov. Int. J. Syst. Evol. Microbiol. 2002, 52, 2141-2146. [CrossRef] [PubMed] 
65. Jensen, T.; Abdelmalek, M.F.; Sullivan, S.; Nadeau, K.J.; Green, M.; Roncal, C.; Nakagawa, T.; Kuwabara, M.; Sato, Y.; Kang, D.-H.; et al. Fructose and sugar: A major mediator of non-alcoholic fatty liver disease. J. Hepatol. 2018, 68, 1063-1075. [CrossRef] [PubMed]

66. Zhao, S.; Jang, C.; Liu, J.; Uehara, K.; Gilbert, M.; Izzo, L.; Zeng, X.; Trefely, S.; Fernandez, S.; Carrer, A.; et al. Dietary fructose feeds hepatic lipogenesis via microbiota-derived acetate. Nature 2020, 579, 586-591. [CrossRef]

67. D’Hennezel, E.; Abubucker, S.; Murphy, L.O.; Cullen, T.W. Total Lipopolysaccharide from the Human Gut Microbiome Silences Toll-Like Receptor Signaling. mSystems 2017, 2, e00046-17. [CrossRef]

68. Le Roy, T.; Llopis, M.; Lepage, P.; Bruneau, A.; Rabot, S.; Bevilacqua, C.; Martin, P.; Philippe, C.; Walker, F.; Bado, A.; et al. Intestinal microbiota determines development of non-alcoholic fatty liver disease in mice. Gut 2013, 62, 1787-1794. [CrossRef]

69. Ley, R.E.; Turnbaugh, P.J.; Klein, S.; Gordon, J.I. Human gut microbes associated with obesity. Nature 2006, 444, 1022-1023. [CrossRef]

70. Zhu, L.; Baker, S.S.; Gill, C.; Liu, W.; Alkhouri, R.; Baker, R.D.; Gill, S.R. Characterization of gut microbiomes in nonalcoholic steatohepatitis (NASH) patients: A connection between endogenous alcohol and NASH. Hepatology 2013, 57, 601-609. [CrossRef]

71. Tortorello, M.L.; Delwiche, E.A. Utilization of fructose and ribose in lipopolysaccharide synthesis by Veillonella parvula. Infect. Immun. 1983, 41, 423-425. [CrossRef]

72. Li, W.; Yang, H.; Zhao, Q.; Wang, X.; Zhang, J.; Zhao, X. Polyphenol-Rich Loquat Fruit Extract Prevents Fructose-Induced Nonalcoholic Fatty Liver Disease by Modulating Glycometabolism, Lipometabolism, Oxidative Stress, Inflammation, Intestinal Barrier, and Gut Microbiota in Mice. J. Agric. Food Chem. 2019, 67, 7726-7737. [CrossRef]

73. Rajilić-Stojanović, M.; De Vos, W.M. The first 1000 cultured species of the human gastrointestinal microbiota. FEMS Microbiol. Rev. 2014, 38, 996-1047. [CrossRef] [PubMed]

74. Wang, K.; Liao, M.; Zhou, N.; Bao, L.; Ma, K.; Zheng, Z.; Wang, Y.; Liu, C.; Wang, W.; Wang, J.; et al. Parabacteroides distasonis Alleviates Obesity and Metabolic Dysfunctions via Production of Succinate and Secondary Bile Acids. Cell Rep. 2019, 26, 222-235.e5. [CrossRef]

75. Salonen, A.; Lahti, L.; Salojärvi, J.; Holtrop, G.; Korpela, K.; Duncan, S.H.; Date, P.; Farquharson, F.; Johnstone, A.M.; Lobley, G.E.; et al. Impact of diet and individual variation on intestinal microbiota composition and fermentation products in obese men. ISME J. 2014, 8, 2218-2230. [CrossRef] [PubMed]

76. Dao, M.C.; Everard, A.; Aron-Wisnewsky, J.; Sokolovska, N.; Prifti, E.; Verger, E.O.; Kayser, B.D.; Levenez, F.; Chilloux, J.; Hoyles, L.; et al. Akkermansia muciniphilaand improved metabolic health during a dietary intervention in obesity: Relationship with gut microbiome richness and ecology. Gut 2016, 65, 426-436. [CrossRef] [PubMed]

77. Martínez, I.; Kim, J.; Duffy, P.R.; Schlegel, V.L.; Walter, J. Resistant Starches Types 2 and 4 Have Differential Effects on the Composition of the Fecal Microbiota in Human Subjects. PLoS ONE 2010, 5, e15046. [CrossRef]

78. Davis, L.M.G.; Martínez, I.; Walter, J.; Goin, C.; Hutkins, R.W. Barcoded Pyrosequencing Reveals That Consumption of Galactooligosaccharides Results in a Highly Specific Bifidogenic Response in Humans. PLoS ONE 2011, 6, e25200. [CrossRef]

79. Mardinoglu, A.; Wu, H.; Bjornson, E.; Zhang, C.; Hakkarainen, A.; Räsänen, S.M.; Lee, S.; Mancina, R.M.; Bergentall, M.; Pietiläinen, K.H.; et al. An Integrated Understanding of the Rapid Metabolic Benefits of a Carbohydrate-Restricted Diet on Hepatic Steatosis in Humans. Cell Metab. 2018, 27, 559-571.e5. [CrossRef]

80. David, L.A.; Maurice, C.F.; Carmody, R.N.; Gootenberg, D.B.; Button, J.E.; Wolfe, B.E.; Ling, A.V.; Devlin, A.S.; Varma, Y.; Fischbach, M.A.; et al. Diet rapidly and reproducibly alters the human gut microbiome. Nature 2014, 505, 559-563. [CrossRef]

Publisher's Note: MDPI stays neutral with regard to jurisdictional claims in published maps and institutional affiliations. 